\title{
A Threshold for Majority in the Context of Aggregating Partial Order Relations
}

\author{
Michaël Rademaker, Member, IEEE and Bernard De Baets
}

\begin{abstract}
We consider a voting problem where voters have expressed their preferences on a single set of objects. These preferences take the shape of strict partial order relations. In order to allow extraction of a unique strict partial order relation corresponding to a social set of preferences, we determine the minimum number of votes a pairwise preference should receive in order to qualify as a social pairwise preference. Transitive closure of the social pairwise preferences will result in the social set of preferences. At the same time, the social set of preferences needs to be cycle-free, and the minimum number of votes should be determined with this constraint in mind. We provide an example application.
\end{abstract}

\section{INTRODUCTION}

We discuss the problem of dealing with a set of preferences in the form of partial order relations, defined on a single set of objects. The aim is to extract or learn a unique richest preference relation from such a set. We have discussed one take on this problem in a previous paper [1], and formulate a new, unrelated approach here. We make no distinction between a set of transitive strict preferences and a multicriteria strict ranking problem, both of which typically result in partial order relations. In some sense, we will think of the partial order relations as voters, and look for the minimum size of the majority for which majority-based voting will yield an outcome that can be transitively closed to yield a strict partial order relation.

The current work can be related to preference learning in machine learning, aiming to learn a ranking function, or a total order relation over the set of objects, on the basis of a set of input pairwise preferences [2], [3]. Additionally, it is clearly related to the field of social choice functions [4], [5], [6], where the concept of majority also plays a crucial role. The work by Vincke [7], containing a number of ranking procedures (a mapping associating a complete pre-order to a relation on a finite set) with a thorough investigation of a number of properties, also involves calculating scores in order to decide on the final (complete or partial) pre-order of the objects under consideration.

\section{Preliminaries}

A (binary) relation $R$ on a set of objects $Q$ denotes some property or characteristic that objects of $Q$ can have w.r.t. each other, i.e. $x R y$ means " $x$ is $R$-related to $y$ ". For example, $R$ could denote "smaller than" or "less polluted than". A relation $R$ on $Q$ can be represented as a set of couples of objects from $Q$; for example $R=\{(a, b),(c, d)\}$

Michaël Rademaker and Bernard De Baets are with the Department of Applied Mathematics, Biometrics and Process Control, Ghent University, Belgium. denotes $a R b$ and $c R d$. If a relation $R$ fulfills the properties of reflexivity $(x R x)$ and transitivity ( $x R y$ and $y R z$ imply $x R z)$, it is called a pre-order relation. If it also has the property of antisymmetry ( $x R y$ and $y R x$ imply $x=y$ ), in addition to reflexivity and transitivity, the relation $R$ constitutes a partial order relation. If $x R y$ or $y R x, x$ and $y$ are commonly said to be comparable. An order relation is strict, often denoted by the symbol $<$, if it is irreflexive $(\neg(x<x))$ and asymmetric $(x<y$ implies $\neg(y<x))$. A couple $(Q,<)$, with $Q$ a set of objects and $<$ a strict order relation on $Q$, is called a (strictly) partially ordered set, or poset for short. We will restrict ourselves to preference relations in the shape of strict partial order relations for both the input and the output preference relations.

We are dealing with a set of objects $Q$ which have been (partially) ordered on the basis of several strict preference relations $R_{i}$. As such, we have $\left(Q, R_{1}\right),\left(Q, R_{2}\right),\left(Q, R_{3}\right)$ and so on. When the preference relations are strict partial order relations, it is natural to write $\left(Q,<_{1}\right),\left(Q,<_{2}\right)$, $\left(Q,<_{3}\right)$ and so on.

A relation $R^{\prime}$ on $Q$ is called an extension of a relation $R$ on $Q$ if it holds that $R \subseteq R^{\prime}$ (it is equivalent to say that $R$ is a subset of $R^{\prime}$ ). The unique smallest transitive extension of $R$ is called the transitive closure of $R$, often denoted as $\widehat{R}$ and commonly computed via the Floyd-Warshall algorithm [8], [9], though other algorithms exist [10], [11]. Similarly, a poset $\left(Q,<^{\prime}\right)$ is called an extension of a poset $(Q,<)$ if $<^{\prime}$ is an extension of $<$. We say that two relations $R_{1}, R_{2}$ on a set $Q$ contradict each other on two objects $x, y \in Q$ if we have $x R_{1} y$ and $y R_{2} x$ (and not $y R_{1} x$ or $x R_{2} y$ ), or $y R_{1} x$ and $x R_{2} y$ (and not $x R_{1} y$ or $y R_{2} x$ ).

In this paper, we will construct partial order relations as a function of a set of partial order relations on a single data set. On the basis of the set $\mathcal{S}$ of partial order relations $R$ we define the function $\mathcal{R}: Q \times Q \rightarrow[0,1]$ as follows:

$$
\mathcal{R}(x, y)=\frac{|\{R \mid R \in \mathcal{S} \wedge x R y\}|}{|\mathcal{S}|} .
$$

We define a threshold $\alpha$ and a relation $\mathcal{R}_{\alpha}$ in this context, where $x \mathcal{R}_{\alpha} y$ holds if $\mathcal{R}(x, y) \geq \alpha$. As such, $\mathcal{R}_{1 /|\mathcal{S}|}$ is the relation denoting that for at least one $R$ from $\mathcal{S}, x R y$ holds. More generally for any $\alpha$, the minimum number of partial order relations from $\mathcal{S}$ that need to contain a specific ordered pair so as to allow that pair to be present in $\mathcal{R}_{\alpha}$, is equal to $\lfloor(\alpha \times|\mathcal{S}|)\rfloor$. One could also think of $\alpha$ as a decision boundary, as all $\mathcal{R}(x, y)$ smaller than this value are not taken into consideration in $\mathcal{R}_{\alpha}$.

In general $\mathcal{R}_{\alpha}$ does not constitute a (pre-)order relation, 
nor does its transitive closure $\hat{\mathcal{R}}_{\alpha}$. We will look for ways to extract such a relation from $\mathcal{S}$ by selecting a suitable $\alpha$ on the basis of the function $\mathcal{R}$. We will show that a minimal $\alpha$ leads to a maximal $\hat{\mathcal{R}}_{\alpha}$, and consider this a way to learn a single set of pairwise preferences over a set of objects. Observe that each relation $R \in \mathcal{S}$ need not voice an opinion on each pair of objects.

\section{MAin Results}

\section{A. Minimizing $\alpha$ Leads to Maximizing $\hat{\mathcal{R}}_{\alpha}$}

Consider $\alpha, \beta \in[0,1]$, with $\alpha<\beta$. Clearly, $\mathcal{R}_{\beta} \subseteq \mathcal{R}_{\alpha}$ by definition: if for an $x, y \in Q \mathcal{R}(x, y) \geq \beta$ holds, then so will $\mathcal{R}(x, y) \geq \alpha$. The same holds for the transitive closures of $\mathcal{R}_{\alpha}$ and $\mathcal{R}_{\beta}$, namely $\hat{\mathcal{R}}_{\beta} \subseteq \hat{\mathcal{R}}_{\alpha}$. As such, if we want to extract a most informative strict partial order relation on the basis of $\mathcal{R}$ or $\mathcal{S}$, we should look for the minimal value for $\alpha$ for which $\hat{\mathcal{R}}_{\alpha}$ is a strict partial order relation. We will denote this minimum value as $\alpha_{\min }$.

\section{B. Computing $\hat{\mathcal{R}}_{\alpha}$}

As mentioned in Section II, algorithms exist to compute the transitive closure of any relation, in our case $\mathcal{R}_{\alpha}$. If, during the closing procedure, we would encounter a cycle (for example, we have $x \mathcal{R}_{\alpha} y$ and $y \mathcal{R}_{\alpha} z$, yielding $x \hat{\mathcal{R}}_{\alpha} z$, while we already have $z \mathcal{R}_{\alpha} x$ or ascertained $z \hat{\mathcal{R}}_{\alpha} x$ ), we can halt the procedure and conclude $\hat{\mathcal{R}}_{\alpha}$ will not be a partial order relation. Obviously, each check to see if $\mathcal{R}_{\alpha}$ can be closed to yield a partial order relation, is at most of the same complexity (in the number of elements of the posets) as the transitive closure operation, i.e. $\mathcal{O}\left(|R|^{3}\right)$.

\section{Upper and Lower Bounds to $\alpha_{\min }$}

In effect, we are dealing with a voting problem, with $|\mathcal{S}|$ voters, each of whom entered a partial order relation in the form of a list of transitive pairwise preferences. Based on these voters, we want to determine a strict partial order relation, which by definition cannot contain cycles. From social choice and voting theory, we know that the regular concept of pairwise majority does not preclude existence of a cycle. Hence, a value of $\frac{1}{2}$ will not be an upper bound for $\alpha_{\min }$. In contrast, a lower bound for $\alpha_{\min }$ is easily found on the basis of $\mathcal{R}$. This lower bound is exactly equal to

$$
\frac{1}{|\mathcal{S}|}+\max _{x, y}(\min (\mathcal{R}(x, y), \mathcal{R}(y, x))),
$$

which can at most be equal to $\frac{1}{|\mathcal{S}|}+\frac{1}{2}$. If $\alpha$ were lower than the value in (2), we would immediately have $x \mathcal{R}_{\alpha} y$ and $y \mathcal{R}_{\alpha} x$ for at least one $x \neq y$. Consequently, $\hat{\mathcal{R}}_{\alpha}$ would then definitely not be a partial order relation.

A trivial upper bound for $\alpha_{\min }$ is 1 , which simply states that if we only include those relations which are present in all $R \in \mathcal{S}, \hat{\mathcal{R}}_{\alpha}$ (in this case equal to $\mathcal{R}_{\alpha}$ ) will be a partial order relation. This amounts to simply taking the intersection of all partial order relations $R \in \mathcal{S}$.

\section{Efficient Determination of $\alpha_{\min }$}

The number of different values that $\mathcal{R}(x, y)$ can take, is the number of partial order relations in $\mathcal{S}$ plus 1 . We do not need to examine all $|\mathcal{S}|+1$ possible values for $\alpha$ however. We know that if for a given $\alpha, \hat{\mathcal{R}}_{\alpha}$ is not a strict partial order relation, then no $\hat{\mathcal{R}}_{\beta}$, with $\beta>\alpha$, can be a strict partial order relation as well. Consequently, we will need at most $\log _{2}(|\mathcal{S}|+1)$ steps in order to determine $\alpha_{\text {min }}$. We are now able to construct an algorithm to compute $\alpha_{\min }$ and the corresponding $\hat{\mathcal{R}}_{\alpha_{\min }}$ (often, we will simply write $\hat{\mathcal{R}}$ when no confusion is possible).

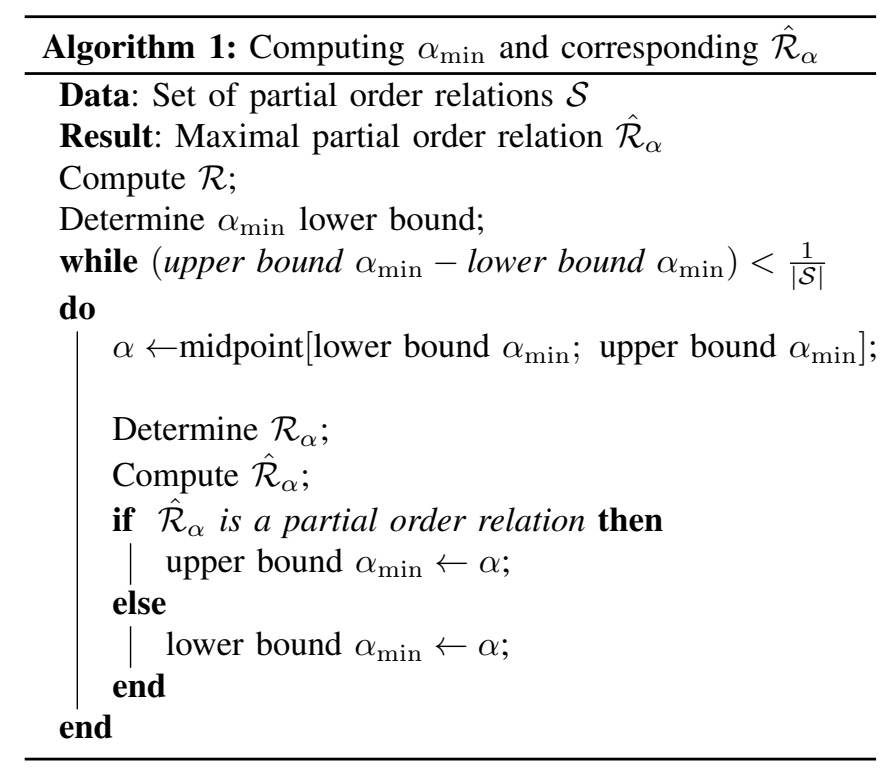

A better way to determine $\alpha_{\min }$ could be to construct a relation $\mathrm{R}: Q \times Q \rightarrow[0,1]$, and initialize each $\mathrm{R}(i, j)$ by setting it to the corresponding $\mathcal{R}(x, y)$ value. Subsequently, the transitive closure of this relation could be performed, denoted as $\hat{R}$, or by iteratively setting

$$
\mathrm{R}(i, j)=\max \left\{\mathrm{R}(i, j), \max _{k \in Q}\{\min \{\mathrm{R}(i, k), \mathrm{R}(k, j)\}\}\right\},
$$

and repeating this operation until no $\mathrm{R}(i, j)$ changes (requiring at most $|Q|$ iterations). Once this relation is constructed, $\max (\mathrm{R}(x, y) \mid \mathrm{R}(x, y) \leq \mathrm{R}(y, x))$ immediately yields $\alpha_{\min }$. In fact, letting $\mathcal{R}$ correspond to those $\hat{\mathrm{R}}(i, j)>\alpha_{\min }$ would immediately yield the desired partial order relation $\hat{\mathcal{R}}_{\alpha_{\min }}$. This results in the much simpler Algorithm 2.

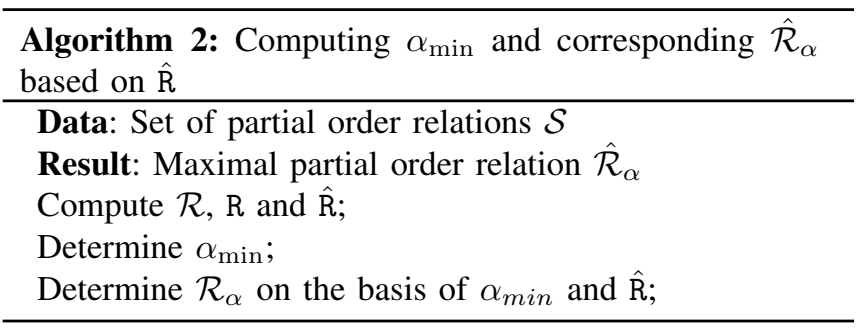

The two algorithms are equivalent as for an $\alpha$ in between $\mathcal{R}(i, j)$ and $\mathrm{R}(i, j), i \mathcal{R} j$ would be induced by transitivity. 
Thus, selecting $\alpha_{\min }$ on the basis of $\mathrm{R}$ values is equivalent to selecting an $\alpha_{\min }$ on the basis of the $\mathcal{R}$ values taking the subsequent transitive closure of the resulting $\mathcal{R}_{\alpha}$ in mind.

\section{E. Interpretation of $\alpha_{\min }$}

If $\alpha_{\min }$ takes a low value, one can surmise the sets of preferences to complement one another, while a high value of $\alpha_{\min }$ indicates that the sets of preferences contradict one another on at least one relation, or would exhibit a cyclical preference relation. Two partial order relations of the same size can thus be compared on the basis of their $\alpha_{\min }$ value. On the other hand, the number of relations in $\mathcal{R}_{\alpha_{\min }}$ compared to $\mathcal{R}_{0}$ could also be a measure of the extent to which the partial order relations contradict rather than complement each other.

\section{F. Subsets of $\mathcal{S}$ - Robustness to Noisy or Non-Informative Preference Relations}

Suppose we are puzzled by the high value of an $\alpha_{\min }$ and suspect one of the sets of pairwise preferences to be incorrect or a protest vote. To what extent can such a set disturb the procedure? We first mention that a single set has an influence of at most $\frac{1}{|\mathcal{S}|}$ on each $\mathcal{R}(x, y)$ or $\mathrm{R}(x, y)$ value, and even on the $\hat{\mathrm{R}}(x, y)$ values. As such, a single noisy set of preferences can be expected to cause only few problems, in contrast with accepting only those pairs for which all sets of preferences agree, or with taking the union of all preferences. The intersection commonly results in only very few preference relations being accepts, while the union quickly degenerates into a set weak order relations with extensive equivalence classes, as we illustrated in [1].

Suppose we have a set of preferences $R$ which is, in fact, an empty set: no object is preferred to another object, according to $R$. If we were to add this empty set to $\mathcal{S}$, how would this affect $\hat{\mathcal{R}}_{\alpha_{\min }}$ ? Clearly, each of the non-zero $\mathcal{R}(x, y)$ values would become slightly smaller, by a fraction $1-\frac{|\mathcal{S}|-1}{|\mathcal{S}|}$, as would $\alpha_{\min }$. Nevertheless, $\hat{\mathcal{R}}_{\alpha_{\min }}$ would not be affected: The exact same set of $\mathcal{R}(x, y)$ values would satisfy the new $\alpha_{\min }$ threshold. Obviously, if $S$ is not empty, there can be changes for those pairwise preferences not also part of $S$, even for the objects w.r.t. which $S$ is indifferent.

\section{A PARAmeterized Version of $\mathcal{R}$}

Alternatively, we could devise an $\mathcal{R}: Q \times Q \rightarrow[0,1]$ that makes a distinction between those pairs of objects for which the partial order relations contradict each other, and those pairs for which some of the partial order relations do not express a preference for either object over the other. Let $0 \leq \beta \leq 1 / 2$, and define:

$$
\begin{aligned}
\mathcal{R}^{\beta}(x, y) & =\frac{|\{R \mid R \in \mathcal{S} \wedge x R y\}|}{|\mathcal{S}|} \\
& +\beta \times \frac{|\{R \mid R \in \mathcal{S} \wedge \neg((x R y) \vee(y R x))\}|}{|\mathcal{S}|} .
\end{aligned}
$$

This seemingly minor adjustment has far reaching consequences. First, observe that for $\beta=0$, we retrieve the
TABLE I

$\mathcal{R}, \mathcal{R}^{\prime}, \hat{\mathrm{R}}$ AND $\hat{\mathrm{R}}^{\prime}$ VALUES FOR AN EXAMPLE SET OF PARTIAL ORDER RELATIONS $\mathcal{S}$

\begin{tabular}{c|c|c|c|c}
\hline \hline Couple & $\mathcal{R}$ & $\mathcal{R}^{1 / 2}$ & $\hat{\mathrm{R}}$ & $\hat{\mathrm{R}}^{1 / 2}$ \\
\hline$(a, u)$ & $2 / 5$ & $7 / 10$ & $2 / 5$ & $7 / 10$ \\
$(u, b)$ & $2 / 5$ & $7 / 10$ & $2 / 5$ & $7 / 10$ \\
$(a, v)$ & $2 / 5$ & $4 / 10$ & $2 / 5$ & $4 / 10$ \\
$(v, b)$ & $2 / 5$ & $4 / 10$ & $2 / 5$ & $4 / 10$ \\
$(a, b)$ & $0 / 5$ & $4 / 10$ & $2 / 5$ & $7 / 10$ \\
$(u, v)$ & $0 / 5$ & $5 / 10$ & $2 / 5$ & $6 / 10$ \\
$(u, a)$ & $0 / 5$ & $3 / 10$ & $1 / 5$ & $6 / 10$ \\
$(b, u)$ & $0 / 5$ & $3 / 10$ & $1 / 5$ & $6 / 10$ \\
$(v, a)$ & $3 / 5$ & $6 / 10$ & $3 / 5$ & $6 / 10$ \\
$(b, v)$ & $3 / 5$ & $6 / 10$ & $3 / 5$ & $6 / 10$ \\
$(b, a)$ & $1 / 5$ & $6 / 10$ & $3 / 5$ & $6 / 10$ \\
$(v, u)$ & $0 / 5$ & $5 / 10$ & $2 / 5$ & $6 / 10$ \\
\hline \hline
\end{tabular}

original $\mathcal{R}$. Next, observe that irrespective of the value for $\beta,\left(\mathcal{R}^{\beta}(x, y)-\mathcal{R}(x, y)\right)=\left(\mathcal{R}^{\beta}(y, x)-\mathcal{R}(y, x)\right)$. In other words, $\mathcal{R}(x, y)$ and $\mathcal{R}(y, x)$ would be increased by the exact same amount, and as such, $\mathcal{R}(x, y)<\mathcal{R}(y, x)$ implies $\mathcal{R}^{\beta}(x, y)<\mathcal{R}^{\beta}(y, x)$ for any $\beta$. Nevertheless, it is possible that while we have $y \hat{\mathcal{R}}_{\alpha_{\min }} x$, we have that $x\left(\hat{\mathcal{R}}^{\beta}\right)_{\alpha_{\min }} y$, as we will show below.

Suppose $\beta=1 / 2$ and $|\mathcal{S}|=5$ for a set of 4 objects $a, b, u, v$, with the corresponding $\mathcal{R}, \mathcal{R}^{1 / 2}, \hat{\mathrm{R}}$ and $\hat{\mathrm{R}}^{1 / 2}$ values shown in Table I. The $\hat{\mathrm{R}}$ values have been calculated as mentioned in Section III-D: $\hat{\mathrm{R}}(a, b)$ equals $2 / 5$ because we have $\mathcal{R}(a, u)=\mathcal{R}(u, b)=2 / 5$ (as well as $\mathcal{R}(a, v)=$ $\mathcal{R}(v, b)=2 / 5)$. Clearly, $\hat{\mathrm{R}}(x, y)<\hat{\mathrm{R}}(y, x)$ does not imply $\hat{\mathrm{R}}^{1 / 2}(x, y)<\hat{\mathrm{R}}^{1 / 2}(y, x)$ (see $(a, u)$ and $(u, a),(a, v)$ and $(v, a))$.

Consequently then, it is not guaranteed that the partial order relation $\left(\hat{\mathcal{R}}^{\beta}\right)_{\alpha_{\min }^{\prime}}$ will be an extension of the partial order relation $\hat{\mathcal{R}}_{\alpha_{\min }}$. In fact, we could even have $x\left(\hat{\mathcal{R}}^{\beta}\right)_{\alpha_{\min }^{\prime}} y$ and $y \hat{\mathcal{R}}_{\alpha_{\min }} x$. This is the case in our example. First note that for $\mathcal{R}^{0}$, we have $\alpha_{\min }=3 / 5$ and for $\mathcal{R}^{1 / 2}$, we have $\alpha_{\min }^{\prime}=7 / 10$. For ease of understanding, we go into some more detail: as $\hat{\mathrm{R}}(u, v)=$ $\hat{\mathrm{R}}(v, u)=2 / 5, \alpha$ should be at least $3 / 5$, while $\hat{\mathrm{R}}^{1 / 2}(u, v)=$ $\hat{\mathrm{R}}^{1 / 2}(v, u)=6 / 10$, imply that $\alpha^{\prime}$ should be at least $7 / 10$. These $\alpha_{\text {min }}$ and $\alpha_{\text {min }}^{\prime}$ values lead to $a \hat{\mathcal{R}}_{\alpha_{\min }} u, u \hat{\mathcal{R}}_{\alpha_{\min }} b$ and $b\left(\hat{\mathcal{R}}^{1 / 2}\right)_{\alpha_{\text {min }}^{\prime}} v, v\left(\hat{\mathcal{R}}^{1 / 2}\right)_{\alpha_{\text {min }}^{\prime}} a$. Clearly, we can conclude that $\hat{\mathcal{R}} \nsubseteq \mathcal{R}^{\beta}$ in the general case, and whether or not to use $\mathcal{R}^{\beta}$ rather than $\mathcal{R}$ is open to discussion. Furthermore, while $\mathrm{R}^{1 / 2}$ has the pleasing property that $\mathrm{R}^{1 / 2}(x, y)+\mathrm{R}^{1 / 2}(y, x)=1$, this is no longer the case for $\hat{\mathrm{R}}^{1 / 2}$, as we have for our example $\hat{\mathrm{R}}^{1 / 2}(u, v)+\hat{\mathrm{R}}^{1 / 2}(v, u)=12 / 10$. For other values of $\beta$, similar examples can be found. 


\section{EXAMPLE APPLICATION}

As an illustrative example, we applied $\mathcal{R}^{\beta}$ with $\beta=0$, yielding $\mathcal{R}^{0}$, abbreviated as $\mathcal{R}$ with corresponding $\alpha_{\text {min }}$, and $\beta=1 / 2$, yielding $\mathcal{R}^{1 / 2}$ abbreviated as $\mathcal{R}^{\prime}$ with corresponding $\alpha_{\text {min }}^{\prime}$, to a data set regarding pollution as measured in BadenWürttemberg (BW), a forested region in Germany [12]. More precisely, we consider (in mg per kg of dry matter) the heavy metal pollution in 43 regions in BW, as well as the sulfur deposits. Pollution in these regions has been measured in three vegetation layers, the moss, herb and tree layer. In all layers, lead $(\mathrm{Pb})$, cadmium $(\mathrm{Cd})$ and zinc $(\mathrm{Zn})$ presence have been determined. For the herb and tree layer, the sulfur (S) presence has additionaly been determined.

On the basis of these measurements or indicators, regions can be compared: Two regions are ranked w.r.t. each other if one region is at least as polluted as the other region according to each indicator, and strictly more polluted according to at least one indicator. Without loss of generality, we rank more polluted above less polluted regions.

Using the mentioned indicators, we have constructed five voters or strict partial order relations, on the basis of the following indicators, respectively: (1) the $\mathrm{Pb}, \mathrm{Cd}$ and $\mathrm{Zn}$ pollution in the moss layer; (2) the $\mathrm{Pb}$ and $\mathrm{Cd}$ pollution in the herb layer; (3) the $\mathrm{Zn}$ and $\mathrm{S}$ pollution in the herb layer; (4) the $\mathrm{Pb}$ and $\mathrm{Cd}$ pollution in the tree layer; (5) the $\mathrm{Zn}$ and $\mathrm{S}$ pollution in the tree layer.

The intersection of the five strict partial order relations is empty. Using $\beta=0$ and $\beta=1 / 2$ results in respectively $\alpha_{\text {min }}$ and $\alpha_{\text {min }}^{\prime}$, as well as the corresponding $\hat{\mathcal{R}}_{\alpha_{\text {min }}}$ and $\hat{\mathcal{R}}_{\alpha_{\text {min }}^{\prime}}^{\prime}$. We find an $\alpha_{\min }$ of $3 / 5$ for $\beta=0$, corresponding to a majority. For $\beta=1 / 2$, we find an $\alpha_{\min }^{\prime}$ value of $3.5 / 5$. The corresponding $\hat{\mathcal{R}}_{\alpha_{\min }}$ and $\left(\hat{\mathcal{R}}^{\prime}\right)_{\alpha_{\text {min }}^{\prime}}$ differ a great deal, however, with $\left(\hat{\mathcal{R}}^{\prime}\right)_{\alpha_{\text {min }}^{\prime}}$ comprising 354 pairwise preferences, being an extension of $\hat{\mathcal{R}}_{\alpha_{\min }}$ in this case, comprising 126 pairwise preferences.

By splitting the herb and tree layer pollution indicators in two sets, these layers have twice as much influence as the moss layer pollution. By adding an additional copy of the moss layer poset as a voter for a total of six voters, the resulting $\alpha$ thresholds and partial order relations are slightly different. We find for $\beta=0$ an $\alpha_{\min }$ of $3 / 6$, corresponding to a weak majority. For $\beta=1 / 2$, we find an $\alpha_{\text {min }}^{\prime}$ value of $4 / 6$. The corresponding $\hat{\mathcal{R}}_{\alpha_{\text {min }}}$ for the six voters is almost equal to the $\hat{\mathcal{R}}_{\alpha_{\min }}$ for the five voters: it contains one additional pairwise preference, yielding 127 pairwise preferences instead of 126 . The $\left(\hat{\mathcal{R}}^{\prime}\right)_{\alpha_{\text {min }}^{\prime}}$ partial order relations differ much more, with $\left(\hat{\mathcal{R}}^{\prime}\right)_{\alpha_{\min }^{\prime}}$ for the six voters comprising 373 pairwise preferences, compared to the 354 from before. Furthermore, $\left(\hat{\mathcal{R}}^{\prime}\right)_{\alpha_{\text {min }}^{\prime}}$ for the six voters is no longer an extension of the previous one, although it is close: Of the 354 pairwise preferences in the five voter partial order relation, 352 are also present in the six voter partial order relation.

\section{CONCLUSIONS}

We have described a simple approach to learn a set of pairwise preferences from a given collection of such sets, meaning we are dealing with a social choice procedure. By regarding each such set, or each pairwise preference relation, as a voter, we learned a set of pairwise preferences through a majority-based voting process. We did so by determining the minimum size the majority should take in order to avoid cyclical or contradictory preferences. We have formulated two variants, differing in the way they deal with incomparable objects. Both approaches are easily computed and understood, and will yield a unique solution in each case.

Opportunities for future work are many. First and foremost, the properties of the proposed social choice procedures need to be investigated, in the same manner as performed in e.g. [7]. Secondly, an obvious extension would be to allow non-strict partial order relations as input, though care should be taken not to do so in a naive way: One should assure that a non-strict preference between two objects does not make it more or less likely for either one of the objects to be strictly preferred to the other in the constructed partial order relation. Finally, the interaction with a previous take on this social choice problem, where we determine a consistent union of two possibly contradicting partial order relations [1], merits further investigation. Combining both approaches could allow to not just determine an $\alpha$ for which the resulting $\hat{\mathcal{R}}_{\alpha}$ is a partial order relation, but to determine an $\alpha^{*}<\alpha$ and corresponding $\mathcal{R}_{\alpha^{*}}$ from which a maximal consistent partial order relation can be extracted in the same line as the consistent union operation from [1].

\section{REFERENCES}

[1] M. Rademaker, B. De Baets, and H. De Meyer, "New operations for informative combination of two partial order relations with illustrations on pollution data," Computational Chemistry and High Throughput Screening, vol. 11, pp. 745-755, 2008.

[2] J. FÃGErnkranz and E. HÃCEllermeier, "Pairwise preference learning and ranking," Lecture Notes in Computer Science, vol. 2837, pp. 145156,2003

[3] E. HÃCEllermeier, J. FÃCErnkranz, W. Cheng, and K. Brinker, "Label ranking by learning pairwise preferences," Artificial Intelligence, vol. 172, no. 16-17, pp. 1897-1916, 2008

[4] P. Fishburn, "Social choice functions," SIAM Review, vol. 16, no. 1, pp. 63-90, 1974.

[5] — , "Paradoxes of voting," American Political Science Review, vol. 68 , pp. 537-546, 1974.

[6] — , "Condorcet social choice functions," SIAM Journal on Applied Mathematics, vol. 33, pp. 469-489, 1977.

[7] P. Vincke, "Exploitation of a crisp relation in a ranking problem," Theory and Decision, vol. 32, pp. 221-240, 1992.

[8] R. Floyd, "Algorithm 97: Shortest path," Communications of the ACM, vol. 5, p. 345, 1962.

[9] S. Warshall, "A theorem on boolean matrices," Journal of the ACM, vol. 9, pp. 11-12, 1962.

[10] B. De Baets and H. De Meyer, "On the existence and construction of $T$-transitive closures," Information Sciences, vol. 152, pp. 167-179, 2003.

[11] H. Naessens, H. De Meyer, and B. De Baets, "Algorithms for the computation of T-transitive closures," IEEE Transactions on Fuzzy Systems, vol. 10, pp. 541-551, 2002.

[12] R. Brüggemann, K. Voigt, A. Kaune, S. Pudenz, D. Komossa, and J. Friedrich, "Vergleichende Ökologische Bewertung von Regionen in Baden-Württemberg," GSF - Forschungszentrum fÃCEr Umwelt und Gesundheit, Tech. Rep., 1988, gSF-Bericht 20/98. 